\title{
Protée
}

\section{Approche sémiotique exploratoire des paraboles et des images langagières du Sutra du Lotus}

\section{Anna Ghiglione}

Volume 39, numéro 2, automne 2011

URI : https://id.erudit.org/iderudit/1007167ar

DOI : https://doi.org/10.7202/1007167ar

Aller au sommaire du numéro

Éditeur(s)

Département des arts et lettres - Université du Québec à Chicoutimi

ISSN

0300-3523 (imprimé)

1708-2307 (numérique)

Découvrir la revue

Citer cet article

Ghiglione, A. (2011). Approche sémiotique exploratoire des paraboles et des images langagières du Sutra du Lotus. Protée, 39(2), 45-53.

https://doi.org/10.7202/1007167ar
Résumé de l'article

Cet article s'attache aux paraboles et aux images langagières de la version chinoise du Sütra du Lotus, traduit du sanskrit par Kumärajīva en 406. Il souligne le rôle sémiotique que les métaphores filées revêtent dans cette Écriture en tant que signes évocateurs de la bouddhéité, à savoir d'une dimension qui n'est ni sensible ni intelligible, puisqu'elle suppose la disparition de tous les phénomènes et le dépassement de l’illusion identitaire. En exploitant la notion de semiosis illimitée, l'auteure cherche à comprendre quel type d'herméneutique est suggéré dans le texte à ses récepteurs (les lecteurs, les pratiquants) pour atteindre la vacuité et le salut. L'extinction totale et parfaite de la souffrance entraîne, en effet, la déconstruction ontologique de l'enchaînement causal qui régit non seulement le samssära, mais également les systèmes des signes et des significations. 


\title{
APPROCHE SÉMIOTIQUE EXPLORATOIRE DES PARABOLES ET DES IMAGES LANGAGIÈRES DU SÜTRA DU LOTUS
}

\author{
ANNA GHIGLIONE
}

\section{L'INTÉRÊT DU SŪTRA DU LOTUS POUR LA SÉMIOTIQUE}

Le Sūtra du Lotus de la bonne loi (Saddharmapundarīka Sūtra) compte parmi les textes fondamentaux du bouddhisme mahāyāna (le "grand véhicule»). (Euvre anonyme, sa compilation en sanskrit fut vraisemblablement entreprise aux débuts de l'ère chrétienne, contribuant à fournir une base scripturale à cette diramation de la doctrine indienne qui insiste sur l'universalité du salut. En 286, le Sütra du Lotus fut traduit pour la première fois en chinois par Dharmarakșa; puis en 406, par le célèbre moine Kumārajiva (344-413), originaire de l'Asie centrale. En Chine, sa notoriété atteignit son apogée au sein du courant Tiantai 天 台 (Terrasses célestes). Dans le système classificatoire visant à hiérarchiser la littérature bouddhique que le moine Zhiyi 智顗 (538-597) élabora, ce sūtra occupa en effet une place de premier plan. Sa popularité ne manqua pas de franchir les confins de l'Empire du Milieu pour atteindre la Corée, le Vietnam et surtout le Japon.

Notre travail d'exégèse aura pour objet quelques extraits significatifs de la version chinoise (Miaofa lianhua jing 秒法蓮花經) de Kumārajīva, que nous citerons en empruntant en partie la traduction française de Jean-Noël Robert (1997), tout en travaillant sur l'original ${ }^{1}$.

Les compilateurs de ce texte canonique se démarquent par leur emploi récurrent, intentionnel et conscient d'images langagières, grâce auxquelles ils véhiculent des contenus doctrinaux plus abstraits. En d'autres mots, ils exploitent une profusion de métaphores, d'analogies, de comparaisons, d'allégories, de paraboles, c'est-à-dire d'images «qui relèvent de l'activité linguistique et dont le support est un système articulé de signes, phonétiques et graphiques, capables d'exprimer et de communiquer économiquement les contenus de la pensée» (Wunenburger, 2001: 39). Dans cet article, il importera alors d'étudier ce système d'images évocatrices et suggestives, puisqu'elles introduisent «dans la sphère des signes généralement abstraits et arbitraires une dimension concrète, sensible, affective, émotionnelle, poétique voire cosmique» $(\text { ibid. })^{2}$. C'est dans ce sens que cette contribution trouve sa place dans une réflexion d'ordre général et exploratoire sur les liens entre le bouddhisme et la sémiotique. Parmi les procédures de substitution sémantique, la métaphore filée - figure constitutive des allégories et des paraboles - est un fait discursif qui intéresse de près les sémioticiens (Greimas et Courtés, 1979: 227). Or, dans le Sūtra du Lotus, la métaphore filée est à l'honneur; mode de communication indirecte par excellence, elle permet de 
transmettre des vérités doctrinales, autrement indicibles, par un effort d'adéquation au niveau, relativement peu élevé, de maturité spirituelle des destinataires du message bouddhique.

Le Sūtra du Lotus a déjà éveillé notre intérêt dans une étude consacrée au thème de la simulation et des «expédients salvifiques» (sk.: upāya, ch.: fangbian 方 便) ${ }^{3}$ dans le bouddhisme (Ghiglione, 2009a). En bref, il s'agissait de montrer que, dans la diffusion de l'enseignement de la doctrine du Bouddha, la justification morale de l'emploi de méthodes habiles, ayant recours à la tromperie, découle de la déconstruction ontologique radicale opérée par les théoriciens du "grand véhicule» ${ }^{4}$. Le principe de la double vacuité (ch. : er kong 二 空) - selon lequel non seulement le noyau constitutif de l'être humain, l'âme, le soi ou le moi (sk.: ātman; ch.: wo 我), relève de l'ordre de l'illusion (sk.: māya ; ch.: huan 幻), mais les phénomènes (sk.: dharma; ch.: shi 事) aussi sont dépourvus de toute substantialité, d'une nature propre et permanente - amène en effet à dépasser la dichotomie, que la morale grégaire pose, entre simulation et authenticité, sans pour autant aboutir à une position d'indifférenciation éthique. De l'article que nous avons publié autour de cette problématique, il convient de retenir ici une idée importante: les mots et les récits, avec leurs images langagières, sont caractérisés, dans le Sütra du Lotus, comme des moyens habiles et provisoires qui facilitent le chemin de l'éveil à ceux qui doivent encore franchir les limites des vérités ordinaires (Ghiglione, 2009a: 291) ${ }^{5}$.

Le Sūtra du Lotus, étant l'une des Écritures parmi les plus influentes de la tradition du "grand véhicule», a fait l'objet de nombreuses études et commentaires, dont il serait impossible de fournir ici une liste exhaustive. Aussi nous bornerons-nous à quelques références pertinentes pour notre analyse.

L'importance de l'imagerie mentale que ce texte déclenche de par sa simple lecture a attiré l'attention d'Eugene Y. Wang, qui en a étudié le lien avec la culture visuelle de la Chine médiévale. Il remarque dans son introduction:

En tant que "religion des images", le bouddhisme exploite largement les visions, l'optique, la fantasmagorie et la méditation, en dissolvant rapidement les confins entre observateur et observé; il n'est pas dépourvu d'une culture visuelle. (2005: XIX; notre traduction) ${ }^{6}$

En plus des images langagières, il est évident que le Sūtra du Lotus brille par son emploi récurrent de procédés énonciatifs, telle l'hypotypose, consistant à créer un effet de réel 7 . En somme, suivant la philosophie de cette Écriture, le salut, la compréhension de la vérité ultime ou la gnose s'actualisent à travers une expérience foncièrement visuelle ou visionnaire. Les images langagières et les figures de pensée, caractéristiques d'un style imagé, constituent des indices de sens transcendant l'activité dualiste intellective et, par voie de conséquence, l'illusion identitaire, l'impression trompeuse d'une séparation entre sujet et objet.

La place primordiale de la parabole et de l'allégorie dans la littérature bouddhique n'a pas manqué de susciter l'intérêt des philologues. L.N. Menśikov, par exemple, y consacre un article qui fait entre autres allusion au Sütra du Lotus; l'orientaliste russe observe que la parabole constitue un moyen d'éviter le danger "de révéler immédiatement au profane la loi ésotérique du Bouddha dans toute sa profondeur", qui demeure ainsi lettre morte pour les non-initiés (1980: 304).

Pour terminer cette brève revue de la littérature, il convient encore de signaler le riche ouvrage de Michael Pye (2003, en particulier le chap.3) sur les moyens habiles dans la tradition du mahāyāna; l'auteur y mentionne plusieurs paraboles tirées du Sūtra $d u$ Lotus, en les interprétant à la lumière de son explication des expédients salvifiques.

Donc, pour ainsi dire, notre article ne constitue qu'une goutte d'eau dans le vaste océan de la bouddhologie. Son originalité, si originalité il y a, est foncièrement contextuelle: faisant partie intégrante d'un dossier qui s'attache au bouddhisme et à la sémiotique, il espère livrer aux spécialistes qui étudient les systèmes de signes et de significations des données philologiques utiles à l'avancement de leurs recherches.

BREF APERÇU DES PARABOlES DU SŨTRA DU LOTUS

Le Sütra du Lotus comporte, en plus d'une myriade d'images langagières indépendantes, qui apparaissent 
en dehors d'un récit précis, sept paraboles principales, énoncées d'abord en prose, puis reprises en stances avec un certain nombre de variantes. Autour de ces récits s'articulent sept chapitres différents.

Le chapitre III, intitulé justement «La parabole (Piyu 譬 喻)》, raconte la célèbre histoire de la maison en flammes, qui métaphorise les trois mondes impermanents tels que les êtres animés les connaissent ${ }^{8}$. Le Bouddha y apparaît comme un père empli de compassion (da ci da bei 大慈大 悲, MFLHJ: 13), préoccupé de sauver ses enfants insouciants du feu destructeur. Dans le chapitre suivant - «Croire et comprendre (Xin jie 信 解)»-, l'Éveillé joue un rôle analogue: il porte les habits d'un père riche, généreux et bienveillant envers son fils qui, ayant abandonné la maison parentale pour se livrer à une vie d'errance, avait été réduit à la misère.

«La parabole des plantes médicinales (Yao cao yu 藥草 喻)» titre le chapitre V. L'enseignement du Bouddha est comparé à une pluie bénéfique qui, provenant d'une grande et dense nuée, se répand partout sur le sol, fertilisant les diverses espèces végétales, chacune en fonction de sa nature et de son état.

«La parabole de la ville fantasmagorique (Hua cheng yu 化城喻)» illustre, dans le chapitre VII, l'idée selon laquelle la voie vers la délivrance est longue et difficile. L'Ainsi-Venu (épithète du Bouddha) soulage parfois «les voyageurs» découragés en cours de route à l'aide de stratagèmes. Le texte invite à imaginer que, afin de leur redonner de l'ardeur, l'Éveillé fasse apparaître une grande ville, où les voyageurs puissent faire halte et reprendre leurs forces.

Dans les chapitres VIII et XIV, le précieux enseignement du Bouddha, et celui du Sūtra du Lotus en particulier, est métaphorisé par l'image de la perle cachée respectivement dans la doublure d'un vêtement et dans un chignon. Dans le premier cas, c'est encore un voyageur qui est mis en scène: contraint à une vie d'efforts et de peines, ce personnage égaré ignore que l'un de ses amis lui avait cousu dans son habit une perle d'une valeur inestimable. L'autre récit raconte l'histoire d'un grand roi qui, pour récompenser ses armées de leurs exploits militaires, leur distribue des biens rares et précieux, mais hésite à leur accorder la perle limpide qu'il porte secrètement dans son chignon ${ }^{9}$.

L'Éveillé interprète de nouveau un rôle paternel dans la parabole des enfants empoisonnés. Dans le chapitre XVI, ce récit illustre une fois de plus la nécessité d'avoir recours à des méthodes habiles pour sauver l'humanité qui souffre. Le protagoniste, un médecin, père d'une nombreuse progéniture, se voit obligé de feindre la mort pour convaincre certains de ses fils récalcitrants à absorber le remède efficace qu'il avait préparé. Leur guérison ne se produit que grâce à cet expédient salvifique.

L'utilité d'exposer la doctrine bouddhique à l'aide de figures, de paraboles et d'autres procédés discursifs est clairement défendue dans le chapitre II du sūtra, qui s'attache à la notion d'expédient salvifique:

«Grâce aux relations, paraboles/ et locutions, à la puissance de leurs expédients,/ [les Éveillés] font exulter tous les êtres" (MFLHJ: 7) ${ }^{10}$.

L'expression chinoise qu'on traduit généralement par «parabole» est piyu 譬 喻, où pi signifie «comparer, illustrer par des exemples» et $y u$, «explication, locution». Dans le titre du chapitre III et tout au long du sūtra, ce mot dissyllabique figure dans sa forme complète, alors qu'il est abrégé en $y u$ dans le titre des chapitres V et VII. Par ailleurs, pi et yu ne forment pas toujours une expression binomiale, mais, dans certains cas, il est légitime de les interpréter comme deux termes juxtaposés aux sens (légèrement) différents. Puisque les catégories de la rhétorique occidentale n'ont pas d'équivalents lexicaux exacts dans la rhétorique chinoise, piyu, pi et yu peuvent également indiquer la métaphore, l'allégorie et la comparaison, à savoir des procédés qui ont tous en commun la construction d'un rapport d'analogie entre deux termes (deux signifiés) ${ }^{11}$. Ces oscillations de sens en chinois autorisent ainsi une certaine fluctuation lexicale en ce qui concerne l'exégèse et la traduction en français.

Sur le plan philologique, le Sūtra du Lotus pose des problèmes de paternité et de datation. Même s'il ne peut pas être considéré comme un texte unitaire, issu d'un seul auteur, l'emploi récurrent d'images langagières et de paraboles apparaît comme un vecteur de cohésion et d'organisation textuelle. Les sections 
où figurent les récits susmentionnés dateraient approximativement de l'an 100 de notre ère (Robert, 1997: 12) et appartiendraient ainsi à la deuxième phase de la rédaction de l'œuvre (le prologue et le deuxième chapitre en constitueraient le noyau le plus ancien). Elles se prêtent bien à une lecture qui s'inscrit dans la sémiotique, puisque cette dernière, plutôt que de démembrer le texte en unités distinctes,

[...] part du postulat qu'à une unité donnée de discours (ce texte analysé) correspond une totalité organisée de signification - un micro-univers sémantique - régie par une forme propre [...].

(Larangé, 2009: 34)

Or, ce sont les figures de sens et de pensée qui structurent le Sütra du Lotus, en limitant ainsi les effets de pulvérisation textuelle découlant d'une compilation anonyme, rédigée par plusieurs auteurs à des dates différentes. Pour emprunter les concepts opératoires de Gérard Genette (1972), c'est donc ici le "discours" (la manière de narrer le récit) qui donne une cohérence au récit (le narré) ${ }^{12}$.

\section{DES SIGNES RÉVÉLATEURS AU RÉCIT PARABOLIQUE}

D'une manière générale, l'expérience d'élévation spirituelle dans le bouddhisme, plutôt que de se présenter comme un acte de foi, prend les formes d'une prise de conscience, d'un dépassement de l'ignorance (sk. avidyā) menant à l'éveil (Ghiglione, 2009b: 70). L'ignorance, qui conduit les noninitiés à considérer à tort comme réel le monde phénoménal et à s'y attacher, se dissipe entre autres à travers l'intuition mystique du sens profond des signes révélateurs de la bouddhéité. La notion de xiang 相 constitue la clé de voûte de la sémiotique bouddhique. Ce terme chinois, souvent employé pour rendre le sanskrit dvātriṃ̧advāra-lakșaṇa, se réfère d'abord aux trente-deux signes distinctifs majeurs du Bouddha, dans son hypostase de «souverain universel» (sk. cakravartin; ch.: zhuan lun wang 轉 輪 王), auxquels s'ajoutent quatre-vingts signes mineurs ${ }^{13}$. Trente de ces marques principales, largement exploitées dans l'iconographie, sont de nature visuelle et se révèlent au regard; la vingt et unième fait référence à la subtilité de goût et la vingtcinquième - une voix mélodieuse - sollicite l'ouie.
Dans le Sūtra du Lotus, le terme xiang 相 revêt aussi la signification plus large d'indice pertinent, de signe sensible d'une dimension transcendante, qui dépasse la condition humaine ordinaire. Dans le chapitre VII, ce mot clé fait référence à la manifestation par excellence de la bouddhéité: l'illumination, la lumière mystique, l'éclat des êtres éveillés ${ }^{14}$. Puisque le Vénéré du monde (autre appellation du Bouddha) est fort difficile à voir (shen nan jian 甚 難 見), il convient de rechercher (qiu 求) les signes par lesquels il annonce sa venue. Ainsi, Śikhin, un grand souverain des dieux brahmaniques, adresse à la foule cette question rhétorique en stances:

Pour quelle raison, à présent,/ nos différents palais/ sont-ils

illuminés de l'éclat irradiant d'une majestueuse vertu/ et se trouvent-ils parés de façon inouïe?/ Un signe aussi prodigieux (miao xiang 妙 相)/ ne s'est de par le passé ni entendu ni vu./ Est-ce qu'un dieu aux grandes vertus est né,/ ou est-ce qu'un Éveillé a émergé du monde?

(MFLHJ : 24; trad. modifiée de Robert, 1997: 177)

L'intense lumière qui brille dans les demeures divines annonce l'épiphanie du Bouddha, assis sur son siège léonin. Ce signe révélateur est qualifié de miao, terme qui dans les Écritures bouddhiques (et taoïstes) chinoises désigne justement le prodigieux, le surnaturel, voire les mystères inaccessibles à la raison et à la perception de l'homme du commun. Il est opportun de souligner que le même terme miao qualifie la loi bouddhique ( $f a$ ) dans le titre du Sütra en question, qu'il conviendrait alors de rendre par Le Sütra de la loi prodigieuse, comme le font certaines traductions anglaises (voir Soothill, 1930).

Or, les images langagières, les métaphores constitutives des paraboles et des allégories qui figurent dans cette Écriture jouent une fonction analogue, sur le plan discursif, à celle des signes pertinents (xiang) qui sont censés préparer les profanes à l'éclosion d'une vérité disruptive, susceptible de transformer radicalement leur représentation du monde et leur sensibilité. Le rôle des images langagières est, somme toute, ambivalent: en tant que modes de communication indirecte, elles diffèrent l'instant de l'intuition de la vérité ultime, qui suppose alors une démarche herméneutique, de type 
anagogique, menant à la gnose; en tant que méthodes habiles, elles revêtent une fonction pédagogique et représentent une propédeutique indispensable au développement d'un esprit d'éveil.

L'interprétation des paraboles, par ailleurs, ne se veut ni libre ni interminable: le texte se suffit à luimême en ce qu'il suggère des clés de lecture, des pistes de réflexion accompagnant le récepteur (le lecteur, le pratiquant) vers la découverte du sens, généralement univoque, du récit. Dans le chapitre VII, l'Ainsi-Venu est présenté explicitement comme un grand guide ( $d a$ dao shi 大 導 師), qui énonce la parabole de la ville fantasmagorique et oriente la communauté vers la juste compréhension de sa signification. Ainsi, d'une certaine manière, le sūtra fournit aux récepteurs un appareil sémiologique leur permettant de décoder la logique de plusieurs sous-ensembles de signes linguistiques, intentionnellement utilisés à des fins communicatives ${ }^{15}$. L'impression d'hermétisme que les paroles du Bouddha suscitent dans un premier temps se dissipe au fur et à mesure que l'on avance dans l'écoute ou dans la lecture du texte.

Selon la sotériologie sous-jacente au Sūtra du Lotus, l'Éveillé possède des pouvoirs surnaturels (shen li 神 力) et une sagesse (zhi hui 智 慧) extraordinaire qui, justement en raison de leur perfection, ne peuvent être communiqués à des êtres encore soumis aux souffrances du samsāra (MFLHJ : 13). Il est acquis que le recours aux expédients salvifiques, dont la parabole, comble cet écart, cette différence de niveau qui sépare les ignorants des esprits éclairés, en rendant possible la communication. Les stratagèmes langagiers employés dans un but salvifique forment alors un véritable code comportant ses règles d'interprétation. Les récepteurs du message, tout en étant orientés vers un décodage précis du récit, ne demeurent pas passifs face à l'émetteur. Ils quittent progressivement le plan du sens littéral pour atteindre celui du sens figuré, passage qui coïncide bel et bien avec une expérience de sublimation spirituelle.

Force est de constater que cette Écriture présente la «culture» de l'esprit d'éveil comme une démarche graduelle (jian 漸) et progressive en ce qu'elle demande d'endurer des efforts souvent pénibles avant d'arriver à maturité ${ }^{16}$. La route est escarpée, les voyageurs sont enclins à des phases de découragement et de lassitude: des étapes intermédiaires fantasmagoriques s'avèrent alors bénéfiques dans la quête du salut.

Dans le chapitre IV, où figure la parabole de l'enfant pauvre, la prise de conscience est délibérément retardée par le père riche qui, après des décennies de séparation, décide de ne pas se laisser reconnaître par son fils en prononçant une déclaration explicite. Il ordonne plutôt à ses subalternes:

"Je n'ai nul besoin de cet homme, ne le forcez pas à venir. Versez-lui de l'eau froide sur le visage pour qu'il reprenne conscience (xing wu 醒 悟) ${ }^{17}$, mais ne lui adressez plus la parole». Pourquoi cela? Le père avait compris (zhi 知) que l'inclination d'esprit de son fils était vile; il avait compris (zhi) que sa propre richesse était pour lui un obstacle, et il avait parfaitement compris (zhi) que c'était son fils, mais, en manière d'expédient, il ne déclara (yu 語) pas aux autres: "C'est mon fils».

(MFLHJ: 17; trad. modifiée de Robert, 1997: 130)

Il convient de noter que le mot wu, dans la locution xing wu que nous avons rendue par «reprendre conscience», signifie aussi «éveil», au sens mystique du terme. Or, il y a éveil et éveil. Un jet d'eau froide permet certes de se reprendre physiquement sur le coup, mais le salut ne s'obtient qu'au prix d'un travail sur soi long et douloureux. Afin de se préparer à un acte final de reconnaissance officielle, le fils sera alors employé par son propre père comme humble déblayeur d'immondices pendant au moins vingt ans. Le récit joue sur un double registre: d'une part, la compréhension du stratagème du père n'est pas entièrement laissée au jugement du fils; d'autre part, l'interprétation de la parabole n'est pas complètement confiée à la perspicacité des récepteurs. Le passage du littéral au figuré s'actualise en créant une sorte de mise en abyme, puisque la parabole suggère les modalités de sa propre herméneutique: une intériorisation progressive (jian), réfléchie et guidée de la vérité.

\section{LE SECRET PUBLIC DES ÉVEILLÉS...}

Nous avons expliqué que le Sūtra du Lotus est une Écriture représentative du "grand véhicule»: sa sotériologie accorde une importance capitale à la 
nécessité de répandre l'enseignement bouddhique à l'ensemble des êtres animés. Tout au long du texte, les compilateurs soulignent la supériorité de cette approche, dévalorisant les zélateurs du «petit véhicule", qui ne s'efforceraient pas de donner une portée universelle au salut ${ }^{18}$. Pourtant, le dharma est troublant à bien des égards; sa révélation prématurée se heurterait à des barrières mentales et à des défenses psychologiques rigides, elle déstabiliserait même les dieux et les titans! Aussi l'Éveillé recommande-t-il à son disciple de s'en tenir à la réticence et interrompt-il brusquement son discours:

Cesse, Śäriputra. Si j'exposais ces choses, les dieux, les hommes et les titans de l'ensemble de ces mondes seraient tous en proie à la frayeur (jing 驚) et au doute (yi 疑), les moines outrecuidants tomberaient dans un grand gouffre.

(MFLHJ: 6; trad. modifiée de Robert, 1997 : 73)

Or, dans les textes traditionnels chinois, avant l'adoption de la ponctuation occidentale au XXe siècle, la figure de l'aposiopèse (réticence), au sens strict du terme, n'était pas signalée par des marqueurs précis. Les Écritures et les Classiques chinois sont pourtant réputés pour leur style allusif et indirect et pour leurs sous-entendus fréquents. Dans le Sūtra $d u$ Lotus, le récit parabolique exprime alors, dans toute sa plénitude, cette intention de réticence que le Bouddha manifeste à maintes reprises et en termes tout à fait explicites, frôlant ainsi délibérément l'absurde. Cette dialectique entre le dit et le non-dit suscite alors la complicité du récepteur, qui s'apprête à recevoir les secrets de la Loi prodigieuse et à se mettre à l'unisson avec les Éveillés plutôt qu'avec les noninitiés, incapables de comprendre.

La parabole qui insiste le plus sur l'importance de diffuser le secret du dharma est celle des plantes médicinales (chap. V). L'imaginaire de ce récit est encodé dans une série d'expressions figuratives qui relèvent de l'iconique: le nuage, la pluie, les arbres, les herbes, les céréales, le riz, les cannes à sucre, les vignes, etc. ${ }^{19}$. Suivant la figure de la synecdoque, l'étendue du vivant est représentée par le règne végétal car, d'une manière ou d'une autre, toutes les plantes sont perméables à la pluie. La tension entre secret et révélation filtre encore une fois à travers le voile du langage figuré. D’une part, le Vénéré du monde ne cherche pas à exposer précipitamment (su 速) la Loi, mais «il en tait longtemps la quintessence» (jiu mo si yao 久默斯要; MFLHJ: 19); d'autre part, il l'annonce universellement le moment venu à tous les êtres vivants:

La prédication de l'Éveillé, égale pour tous (ping deng shuo 平等說),/ est comme la pluie, dont la saveur est unique;/ les êtres vivants, selon leur nature,/ ne la reçoivent pas (shou 受) de la même façon,/ tout comme les herbes et les arbres/ sont imprégnés différemment.

(MFLHJ: 20; trad. modifiée de Robert, 1997: 152, 148)

Ainsi, le contenu de l'enseignement bouddhique ne varie pas selon les circonstances, alors que les modalités communicatives de sa diffusion sont multiples et changent en fonction des besoins des récepteurs. Dans cette parabole, le secret concerne la réception du message plutôt que son émission. Le texte est clair sur ce point: la difficulté d'assimiler la doctrine (de se laisser fertiliser par la pluie) tient non pas à une intention d'ésotérisme de la part de l'Éveillé, mais à la disposition d'esprit et à la réceptivité des destinataires.

Mais quel est donc le troublant secret qu'il s'agit de révéler indirectement aux foules, à des êtres aussi nombreux que les sables du Gange? Le texte est constamment elliptique à ce propos, il oriente le lecteur vers l'interprétation du sens univoque de la parabole, qui concerne la phénoménologie de la communication entre l'Éveillé et l'ensemble des êtres vivants, sans pour autant expliciter le contenu précis du secret en question. La philosophie bouddhique, ancrée sur le principe de la vacuité universelle, n'aboutit pas à la construction d'une métaphysique par delà le monde évanescent des phénomènes. Aussi le mystère doctrinal sur lequel elle repose ne suppose-t-il pas une dichotomie entre être et paraître structurant une ontologie. Le secret que les paraboles et les images langagières recèlent n'appartient pas à un domaine interdit, mis à l'écart des entités manifestes; il ne constitue pas «le paraître de quelque être» que les initiés découvriront un jour (Boutang, 1988: 125, 48). Le Bouddha enseigne dans une logique de nondualité qui, en tant que telle, ne vise pas à instaurer 
des inégalités entre les dépositaires des arcanes de sa doctrine et les exclus: «Śāriputra, il te faut savoir que j'ai à l'origine établi un vœu,/ souhaitant rendre l'ensemble des êtres/ égaux à moi, sans différence", déclare-t-il sans crainte (MFLHJ: 8; trad. modifiée de Robert, 1997: 81).

La Loi $(f a)$ sur laquelle règne (wang 王) l'Éveillé est dite «déconstruire l'existence (po you 破 有)», en démontant pièce par pièce la sphère du confectionné et de l'impermanent. Au bout de ce processus d'évidement, tous les différents aspects (xiang, même mot que «signe» en chinois) du dharma convergent vers la vacuité, qui en constitue le seul et unique aspect (MFLHJ: 19), un aspect sans aspect ${ }^{20}$. Rien d'étonnant alors si les prédicateurs retardent délibérément l'herméneutique de leur secret, puisque l'effort interprétatif des initiés conduit non pas à l'Être ou à sa négation, mais à une suspension de sens, à l'annulation de tous les sens singuliers que les vérités ordinaires comportent, pour atteindre, par delà le langage trompeur, le vide sémantique, dont - en vertu d'un ancien oxymore - seul le silence profond des esprits éclairés est l'expression.

\section{RÉFLEXIONS FINALES}

Pour des raisons inhérentes à la philosophie bouddhique, les paraboles du Sūtra du Lotus n'invitent pas les récepteurs à s'égarer dans une herméneutique interminable. Les prédicateurs en surveillent prudemment l'interprétation, énonçant d'abord en prose leurs principes puis les réitérant en stances avec des détails supplémentaires, jusqu'à créer, par cette procédure de répétition, un effet de ritualité, voire de théâtralité - aboutissement maximal de leurs techniques de simulation contingentes. Leurs indications instruisent les foules, des individus les plus ignorants aux auditeurs les plus matures, déclenchant au sein de la collectivité une attitude participative et un sentiment fusionnel libératoire. Dans l'original indien, d'ailleurs, les parties versifiées sont vraisemblablement antérieures aux sections en prose (Robert, 1997: 13-14). L'ajout de ces dernières semble donc répondre, en tout cas, à une exigence de simplification et d'élucidation textuelle: le message de salut mérite qu'on le divulgue.
La sotériologie bouddhique à l'œuvre dans ce sūtra ne saurait orienter les récepteurs vers un phénomène de semiosis illimitée. Il convient de rappeler que ce principe, thématisé entre autres par Umberto Eco (1992: 370), évoque l'idée peircienne selon laquelle: "The meaning of a representation can be nothing but a representation " 21 . Bien au contraire, l'aspiration ultime de l'ascèse bouddhique consiste justement à dissoudre toute représentation mentale, qui ferait obstacle à la délivrance par un alourdissement du flux de l'activité pensante. Dans la même optique, l'utilisation du récit parabolique ne provoque nullement une sorte de "dérive hermétique». Par "l'habileté incontrôlée à glisser de signifié à signifié" (ibid.: 369), une approche pareille différerait en effet à l'infini la compréhension intuitive du texte en déplaçant sans cesse son sens profond et inatteignable. Qui plus est, un livre inspirant à ses interprètes une herméneutique illimitée les condamnerait inéluctablement à la temporalité et, par voie de conséquence, au cycle interminable du saṃāara. Il en va donc tout autrement pour le Sütra du Lotus, dont les indications aident plutôt à franchir les limites spatiotemporelles intrinsèques à l'œuvre même: sa propre texture (espace) et la lecture (temps) relative.

Par ailleurs, le résultat du travail herméneutique que cette Écriture suggère ne saurait trouver davantage sa place dans la finitude d'un système déontologique clos, dominé par quelques dogmes, par des croyances arrêtées, tenues pour vraies une fois pour toutes par des zélateurs à l'esprit borné. La pluie bénéfique qui fertilise le terrain (chap. V), la perle précieuse enfouie dans la doublure du voyageur égaré (chap. VIII), le remède prodigieux susceptible de vaincre le plus fort des poisons (chap. XVI), toutes ces locutions et ces belles images ne sont que «des noms et des graphies fictifs (jia ming zi 假明字)/destinés à guider la multitude des êtres animés" vers la délivrance (MFLHJ: 8; trad. modifiée de Robert, 1997 : 80). Elles indiquent, en guise de subterfuges discursifs, la voie vers l'Extinction, le chemin qui conduit, après la disparition des phénomènes et la dissipation de l'illusion identitaire, à la vacuité. L'extinction de la souffrance, qui suppose celle du sujet et de l'objet, ne peut alors qu'entraîner la cessation totale et parfaite 
de toutes formes de dualité, donc de toutes relations, y compris celles qui régissent les systèmes de signes provisoires et leurs significations, encore imbriquées dans la chaîne causale et temporelle du raisonnement discursif. Le texte canonique n'étant qu'un outil temporaire - une dernière forme de simulation ou fiction -, au bout du processus de sublimation, la semiosis elle-même ne peut que prendre fin.

\section{NOTES}

1. Il existe une autre traduction en français de la même version de ce sütra, réalisée par S. Servan-Schreiber (2007) qui, en réalité, traduit le texte anglais de B. Watson (1993) et non l'original chinois. Par ailleurs, la version sanskrite du sūtra est connue en français grâce à la traduction publiée pour la première fois par E. Burnouf en 1852, travail philologique incontournable. Notre exégèse se base sur le texte chinois de l'édition du Miaofa lianhua jing, publiée sous la direction de J. Takakusu et K. Watanabe [(1925) 1988]; dorénavant MFLHJ). 2. Nous nous prévalons de la caractérisation d'image linguistique proposée par J.J. Wunenburger, avec un point de divergence : le théoricien français exclut les «langues" (selon sa terminologie) non alphabétiques de cette explication, alors que nous la jugeons aussi valable pour l'écriture chinoise, bien qu'elle n'ait jamais atteint le stade du phonétisme pur. À propos de l'analogie et de la métaphore, voir aussi Wunenburger (2002: chap. 2).

3. Dans cet article, les mots clés du bouddhisme sont tantôt indiqués en sanskrit (dorénavant sk.), tantôt en chinois (dorénavant ch.). 4. Sur les aspects ontologiques du mahāyāna, voir P. Magnin (2003: 358).

5. Pour être précise, notre réflexion portait également sur le Sūtra de Vimalakirti, qui date aussi du début de notre ère. Par ailleurs, l'imaginaire sous-jacent à la pensée chinoise prébouddhique, le vaet-vient entre concept et métaphore, la signification philosophique des tropes qui figurent dans les Classiques chinois constituent des objets privilégiés de nos recherches. Voir en particulier nos deux monographies (1999 et 2010)

6. "As a 'religion of images', Buddhism has much use for vision, optics, phantasmagoria, and meditation and readily dissolves the cognitive boundaries between the observer and the observed; it is not short of a visual culture."

7. À propos de ce type de figures de pensée, voir Robrieux (1993: 71). 8. Les six catégories d'êtres animés soumis au cycle des renaissances (le saṃsāra) demeurent dans trois mondes différents: celui du désir, où souffrent entre autres les humains, celui de la forme pure et celui du sans-forme (voir Cornu, 2001 : 627).

9. Dans la version en stances de cette parabole, le monarque finit par défaire son chignon et donner sa perle.

10. Traduction modifiée de J.N. Robert (1997: 79).

11. La question de savoir si la métaphorisation est attestée dans la tradition chinoise fait l'objet de débats animés. Dans notre livre sur la métaphore oculaire dans les Classiques prébouddhiques (Ghiglione, 2010), nous avons montré que la notion de métaphore est pertinente pour décrire plusieurs tropes qui figurent dans les textes chinois de l'Antiquité. Dans l'introduction de cette monographie ( $\$ 2$ en particulier), nous avons aussi abordé la relation entre le sens propre et le sens figuré des mots.

12. Mentionné par Greimas et Courtés (1979: 248).

13. Voir les listes dressées par Cornu (2001: 90-91).

14. Eliade (1957:155) a souligné la signification mystique de la lumière. Voir aussi Ghiglione (2010: 43).

15. À propos de la subtile différence entre sémiotique et sémiologie, voir Coquet (1997: 33).

16. Au VIII siècle dans les milieux bouddhiques, il s'agira de déterminer si l'éveil correspond à une expérience immédiate ou s'il suppose une évolution graduelle. La controverse entre les tenants du subitisme et ceux du gradualisme divisera l'École du Chan. Voir Faure (1988: 168). Malgré l'aspect progressif de la quête du salut que le Sütra $d u$ Lotus affiche, cette œuvre constitue aussi une référence chez les maîtres du zen au Japon.

17. Le fils s'était en effet évanoui, pensant que les assistants de son père voulaient l'emprisonner.

18. Il est utile de rappeler que l'appellation péjorative de «petit véhicule» (hìnayäna) fut forgée par les tenants du "grand véhicule». Les courants qui se rattachent au bouddhisme ancien, précédant la scission du mouvement, se désignent de préférence par le terme «theravāda», qui signifie justement «la voie des anciens".

19. À propos de la distinction entre figuratif iconique et figuratif abstrait dans la parabole, voir Millogo (2007: 191).

20. À propos de la sagesse (wisdom) en tant que compréhension de la nature vide des signes dans le courant du Mādhyamika, voir Bocking et Wang, 2006. Sur la signification doctrinale de l'image du Bouddha qui montre du doigt la lune - une dimension ineffable - dans la tradition Chan, voir Ho, 2008.

21. Citation en anglais dans la traduction française ainsi que dans l'original italien. 


\section{RÉFÉRENCES BIBLIOGRAPHIQUES}

\section{CORPUS ET TRADUCTIONS}

BuRnouf, E. (trad.) [1852]: Le Lotus de la Bonne Loi, Paris, Imprimerie nationale (réimp. en 1973, Paris, Adrien Maisonneuve).

Robert, J.-N. (trad.) [1997]: Le Sûtra du Lotus, Paris, Fayard.

SERVAN-SchreIBER, S. (trad.) [2007]: Le Sûtra du Lotus, Paris, Les Indes

Savantes.

SOOTHILL, W. E. (trad.) [1930]: The Lotus of the Wonderful Law or The

Lotus Gospel, Oxford, Clarendon Press (Abridged translation from the

Chinese of Kumārajīia).

TAKAKUSU, J. et K. WATANABE (dir.) [(1925) 1988]: Taishô shinshû

Daizôkyô (The Tripitaka in Chinese), Meijirodai, Bunkyo-ku Tokyo,

Taisho Shinshu Daizokyo Kanko Kai, Society for the Publication of the

Taisho Tripitaka, vol. 9, 1-62.

WATSON, B. (trad.) [1993]: The Lotus Sutra, New York, Columbia

University Press.

\section{LITTÉRATURE SECONDAIRE}

BOCKING, B. et Y.X. WANG [2006]: «Signs of liberation? A semiotic approach to wisdom in Chinese Madhyamika Buddhism ", Journal of Chinese Philosophy, vol. 33, n³, 376-392.

BOUTANG, P. [(1973) 1988]: Ontologie du secret, Paris, Quadrige/PUF.

COQUET, J.-C. [1997]: La Quête du sens. Le langage en question, Paris,

PUF.

CORnU, P. [2001]: Dictionnaire encyclopédique du bouddhisme, Paris, Seuil. ECO, U. [(1990) 1992]: Les Limites de l'interprétation, Paris, Grasset.

ELIADE, M. [1957]: Mythes, rêves et mystères, Paris, Gallimard.

FAURE, B. [1988]: La Volonté d'orthodoxie dans le bouddhisme chinois,

Paris, Centre national de la recherche scientifique.

GenetTe, G. [(1972) 2007]: Figures III, Paris, Seuil (repris dans Discours du récit, Paris, Seuil).

GHiglione, A. [1999]: La Pensée chinoise ancienne et l'abstraction, Paris, You Feng; [2009a]: «Deception in Chinese Buddhist thinking: Reflections from the Lotus Sütra and the Vimalakìtti Sütra", dans L. Boldt-Irons, C. Federici et E. Virgulti (dir.), Disguise, Deception, Trompe l'œil. Interdisciplinary Perspectives, New York, Washington, Berne, Baltimore, Peter Lang, 285-303;

[2009b]: L'Expérience religieuse en Chine. Sagesse, mysticisme, philosophie, Montréal, Paris, Médiaspaul;

[2010]: La Vision dans l'imaginaire et dans la philosophie de la Chine antique, Paris, You Feng.

Greimas, A. J. et J. COURTÉs [1979]: Sémiotique. Dictionnaire raisonné de la théorie du langage, Paris, Hachette.

Ho, C.-H. [2008]: «The ginger pointing toward the moon: A philosophical analysis of the Chinese Buddhist thought of reference", Journal of Chinese Philosophy, vol. 35, no ${ }^{\circ}, 159-177$.

LARANGÉ, D. S. [2009]: L'Esprit de la lettre. Pour une sémiotique des représentations du spirituel dans la littérature française des XIXe et XXe siècles, Paris, L'Harmattan.

Magnin, P. [2003]: Bouddhisme. Unité et diversité. Expériences de libération, Paris, Cerf.

MENŚIKOV, L.N. [1980]: «Les paraboles bouddhiques dans la littérature chinoise", Bulletin de l'École française d'Extrême-Orient, tome 67, 303-336.

Millogo, L. [2007]: Introduction à la lecture sémiotique, Paris, L'Harmattan.

PYE, M. [(1978) 2003]: Skilful Means: A Concept in Mahayana Buddhism, Londres, New York, Routledge.

ROBRIEUX, J.J. [1993] : Éléments de rhétorique et d'argumentation, Paris, Dunod.

WANG, E. Y. [2005]: Shaping the Lotus Sutra. Buddhist Visual Culture in Medieval China, Seattle, University of Washington Press.

Wunenburger, J.J. [(1997) 2001]: Philosophie des images, Paris, PUF; [2002]: La Vie des images, Grenoble, Presses universitaires de Grenoble. 